\title{
The Characteristics of the Phonetic System of Shigang (Shangping) Dialect in Xinjian District, Nanchang
}

\author{
Jin Shajuan, Xiao Jiugen* \\ Research Center of Language and Language Life, Jiangxi Normal University, Nanchang, China \\ Email address: \\ 2768516469@qq.com (Jin Shajuan),jxsdxjg666666@sina.com (Xiao Jiugen) \\ ${ }^{*}$ Corresponding author
}

To cite this article:

Jin Shajuan, Xiao Jiugen. The Characteristics of the Phonetic System of Shigang (Shangping) Dialect in Xinjian District, Nanchang. Science Innovation. Vol. 8, No. 1, 2020, pp. 11-17. doi: 10.11648/j.si.20200801.15

Received: December 4, 2019; Accepted: March 6, 2020; Published: March 17, 2020

\begin{abstract}
Xinjian District locates in the north of central Jiangxi Province, which belongs to Nanchang Dialect,and it is the representative point of Gan Dialect. Due to it's geographical location and population migration, Nanchang Dialect retains the phonetic features of ancient Chinese relatively completely. However, influenced by the popularization of Mandarin in recent decades, it is more difficult to find the authentic Nanchang Dialect. What's more, some variations in the dialect have arisen. Although the Shigang town(Shangping) Dialect discussed here belongs to the Chang-du group of Gan Dialect and has the common characteristics of the Changdu group, it also has it's own distinctive features. For example, all voiced initial consonants are pronounced as aspirated stop or affricates, regardless of the level and oblique level; the Secondary voiced consonants [n], [1] are not in accordance with the Mandarin, with plum rhyme missed, and with the high and level tone (the first tone of the four Chinese tones) varied.
\end{abstract}

Keywords: Gan Dialect, Shigang Town(Shangping) Dialect, Dialect Characteristics of the Phonetic System

\section{南昌新建区石岗（上坪）的音系特点}

\author{
金莎娟，肖九根 ${ }^{*}$ \\ 江西师范大学语言与语言生活中心, 南昌, 中国 \\ 邮箱 \\ 2768516469@qq. com（金莎娟）, jxsdx jg6666666@sina. com（肖九根） \\ 摘要: 新建区位于赣省中部偏北, 属于赣方言代表点一一南昌方言内。由于其地理位置和人口迁徙等原因, 南昌方言 \\ 较为完整地保留了古汉语的语音特点。不过, 最近几十年受普通话推广的影响, 地道的南昌方言已经越来越难寻了, \\ 且其方言内部也呈现出一定的差异性。此处讨论的石岗 (上坪) 方言虽属赣方言昌都片分区，有着昌都方言区的共性， \\ 但也具自身的个性特点, 如全浊声母不论平入均读送气塞音、塞擦音, 次浊声母 $[\mathrm{n}]$ 、 [ 1 与普通话不对应, 无撮口韵, \\ 阴平有分调等现象，均显示出其独具的鲜明特点。
}

关键词：赣方言，石岗（上坪）方言，方音特点 


\section{1. 引言}

根据赣省境内的内部差别, 学者将赣方言分为南部方 言和北部方言。这两大方言区域的分野, 主要以两个原则 为依据: 一是覃谈非见系分韵, 二是端系合口韵分化成合 口、撮口韵, 或主要元音为圆唇元音。其中, 北部赣方言 分为昌都、乐平、奉新三个片, 南部赣方言分为崇仁、泰 和、分宜、铅山四个片。[1] (第17页) 有的学者还将新建 区方言归为北部昌都片; [2]而新建方言又以望城为界一分 为二一上新建话、下新建话。望城以南的上新建话, 其 代表是松湖、后田、生米和石岗 (上坪) 方言, 具有高安 话的一些特色, 如知彻澄章昌念 $[\mathrm{t}] 、\left[\mathrm{t}^{\mathrm{h}}\right]$ 等; 望城以北的下 新建话, 其代表有望城、大塘、乐化、樵舍和象山, 与永 修、安义话有较多的相似之处, 如声母送气、不送气影响
调类分化等。[3]属于上新建的石岗镇位于新建西南方, 是 南昌、丰城、高安三市交汇点, 锦江河穿镇而过。据2017 年统计, 居民人口约 6.2 万, 面积 133.2 平方公里, 辖 24 个 村委和 1 个居委会, 其地交通密集, 是全镇政治文化的中 心。作为曾经盛极一时的老卫星城, 很多学校、工厂现已 迁回南昌。[4] (第4页) 随着经济的快速发展, 石岗人大 多渐向南昌市新建区政府所在地聚集。受此影响, 石岗 (上 坪）方言也渗透着一定的南昌方言成分, 一定程度上还受 到普通话的同化，这使其处于一个颇具特色的过渡期。

下面是以 《方言调查字表》为蓝本[5](第1-80页) 对 石岗 (上坪) 方言所作的田野调查, 较为全面地反映了其 音系特点。

\section{2. 石岗 (上坪) 方言的语音系统}

\section{1. 声母系统：包括零声母在内, 共有 20 个}
$\mathrm{p}$ 布八兵
$\mathrm{p}^{\mathrm{h}}$ 步别怕平
$\mathrm{m}$ 门麦明
$\mathrm{t}$ 到道夺招蒸
$\mathrm{t}^{\mathrm{h}}$ 太同处虫昌2
$\mathrm{n}$ 你 2
ts 糟祖增争主
$\mathrm{ts}^{\mathrm{h}}$ 仓曹巢潮醋 从粗初除锄
tc 杰精经节结焦举

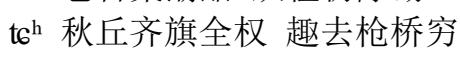
$\mathrm{k}$ 贵系高
$\mathrm{k}^{\mathrm{h}}$ 蹃开葵
ฤ。年认软
y 岸案硬奥
$\mathrm{v}$ 飞符回 ${ }_{2}$

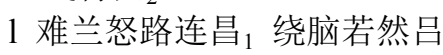
$\mathrm{s}$ 散伞苏书 僧生师诗
6 想手谢响县修 休旋玄线虚系 2
$\mathrm{h}$ 好汗黑

Ø 胡严化话围微午武约言缘元远回 1

（声母系统中, 下标1表白读音, 下标 2 表文读音）。

声母说明:

1) 有唇齿浊擦音 $[\mathrm{v}]$, 发音气流较弱, 上齿与下唇有轻微接触, 只有摩擦受阻, 无送气除阻动作, 如: 付 $\left[\mathrm{vu}^{33}\right] 、 飞\left[\mathrm{vi}^{45}\right]$ 、 副 $\left[\mathrm{vu}^{33}\right]$ 。

2) [n] 的发音舌位靠前。

3) 泥来日母今在洪细音前保持对立。细音前大部分读 $[\mathrm{n}]$, 只有 “你 2 ” 读 $[\mathrm{n}]$, 如: 年 $\left[\mathrm{ni}_{\mathrm{i}} \mathrm{n}^{453}\right]$ 、泥 $\left[\mathrm{n}_{\mathrm{i}}{ }^{453}\right]$; 洪音前读 $[1]$, 如: 脑 $\left[1 \mathrm{lau}^{213}\right]$ 、老 $\left[1 \mathrm{lau}^{213}\right]$ 、热 $\left[1 \mathrm{rt}^{5}\right]$ 。

\section{2. 韵母系统: 共 62 个, 包括自成音节的 $m, n, \quad j$}
1 资知私
$\mathrm{i}$ 第地以雨虚欲倍
u 故鹿奢母
a 爬蛇架
ia 姐野
ua 袜花
$\varepsilon$ 试许那
i $\varepsilon$ 靴鱼
$\mathrm{u} \varepsilon$ 果阔
o 河我坐
uo 过禾

ə 耳二

iu 流收咒

\section{ai 盖介妹}

ei $\square$ (应答声)

$$
\begin{aligned}
& \text { au 饱保桃斗烧 } \\
& \text { an 胆三间含减 } \\
& \text { En 根庚 } \\
& \text { on 酸短 } \\
& \text { on 深争 }{ }_{2} \text { 横 }{ }_{2} \text { 春寸 } \\
& \text { an 硬争 } 1_{1} \\
& \text { on 党桑行讲船床光 } \\
& \text { on 彭朋 }
\end{aligned}
$$

iau 条桥

isn 检连廉权圆

in 紧林心新星 2 云群

ian 星 ${ }_{1}$ 兄 $_{1}$

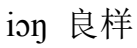

uai 怪帅

uei 碎

ui 鬼桂贵

$$
\begin{aligned}
& \text { uan 关万 } \\
& \text { uen 官管 } \\
& \text { uən 魂温 } \\
& \text { uan 横 }{ }_{1} \text { 梗 } \\
& \text { uoy 翁黄 }
\end{aligned}
$$



$\mathrm{n}^{\mathrm{t}}$ 质室
at 八鸭夹法辣塔
et 不折舌
ot 合割葛
a? 百白只尺
$\varepsilon ?$ 北色直
o? 托落各确
it 急七一吉
iat 洽喫
ict 月接铁缺
i? 踢局
ia? 壁
io? 药削
iu? 六绿
m 姆姆妈
n 你 ${ }_{1}$
五瓦謷
（韵母系统中，符号“口”表示有音无字，下标 1 代表白读音， 2 代表文读音）。
韵母说明:
1) 自成音节有: $[m] 、[n] 、[n]$ 。
2) 入声共有两个韵尾: [?]、[t]，[t]韵尾发音较弱。
3) 没有卷舌元音和儿化音, 与普通话卷舌元音对应的字 读 [ə], [ə]发音开口度稍大, 接近 $[\varepsilon]$ 。
4) 普通话的 [in]韵在石岗 (上坪)方言中读 [in], 如: “斤、 精”不分, 都读作 $\left[\mathrm{tcin}^{45}\right]$ 。

iun 龙 ${ }_{1}$ 兄 $_{2}$ 穷琼胸

\section{3. 声调系统: 除轻声外, 有 7 个调类、 8 个调值}

表1 声调类型。

\begin{tabular}{|c|c|c|}
\hline 调类 & 调值 & 例字 \\
\hline \multirow[b]{2}{*}{ 阴平 } & 甲类 45 & 高猪专尊低边安秃 \\
\hline & $\begin{array}{l}\text { 乙类 } \\
445\end{array}$ & 开抽初粗天偏婚伤三飞 \\
\hline 阳平 & 453 & 穷陈床才唐平寒神徐扶鹅娘人龙难麻文云曲 \\
\hline 上声 & 213 & $\begin{array}{l}\text { 古展纸走短比碗口丑楚草体普好手死粉五女染 } \\
\text { 老暖买网有匹 }\end{array}$ \\
\hline 阴去 & 33 & $\begin{array}{l}\text { 盖账正醉对变爱抗唱菜怕汉世送放岸让漏怒帽 } \\
\text { 望用 }\end{array}$ \\
\hline 阳去 & 11 & $\begin{array}{l}\text { 近柱是坐淡抱厚社似父共阵助贱大病害树谢饭 } \\
\text { 局 }\end{array}$ \\
\hline 阴入 & 5 & $\begin{array}{l}\text { 急竹织积得笔一出七黑湿锡福割桌窄接搭百约 } \\
\text { 缺尺切铁拍歇说削发 }\end{array}$ \\
\hline 阳入 & 1 & 宅食杂读白合舌俗服 \\
\hline
\end{tabular}

声调说明:

1) 古全清平和次清平由声母是否送气而分调, [6](第 188-211页) 将其分为阴平甲、乙二类，且属高升调， 阴平乙类调值前半段平调动程较长，后半段上升。

2) 古全浊上声今归为阳去。

3) 入声分阴阳, 阴入调值高, 阳入调值低。[7]

4) 去声多为平调, 阴去为中平调, 阳去为低平调。

\section{3. 石岗 (上坪) 方言音韵特点}

\section{1. 声母特点}

1) 石岗 (上坪) 方言晓匣母合口字和非组大部分字读唇 齿浊擦音 [v], [8](第22-27页)如: 火[vo213]、花[va45]、 un 东动龙

ut 突

uat 滑刮

uct 活

u? 哭木

ua? 划画

uع? 国

uo? 郭或 飞[vi45]、万[van33], 但发音气流较弱, 微母字部分

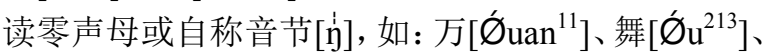
冯 $\left[\mathfrak{y}^{453}\right]$ 、蜂 $\left[i^{45}\right]$ 。

2) 古全浊声母今不论平大一律读送气的塞音、塞擦音, [9]如：步 $\left[p^{\mathrm{h}} \mathrm{u}^{11}\right]$ 、菩 $\left[\mathrm{p}^{\mathrm{h}} \mathrm{u}^{453}\right]$ 、赔 $\left[\mathrm{p}^{\mathrm{h}} \mathrm{ai}{ }^{453}\right]$ 。

3) 古知章庄组今不论开合大部分合流, 只有一组舌尖音, $[\mathrm{ts}] 、\left[\mathrm{ts}^{\mathrm{h}}\right] 、[\mathrm{~s}]$ 一律读作 $[\mathrm{ts}] 、\left[\mathrm{ts}^{\mathrm{h}}\right] 、[\mathrm{~s}]$, 如: 争 ${ }_{1}\left[\mathrm{tsan}^{45}\right]$ 、

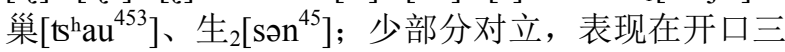
等知章组字保留古端组音的特征, 如: 招 $\left[\mathrm{tau}^{45}\right]$ 、车 $\left[\mathrm{t}^{\mathrm{h}} \mathrm{a}^{445}\right]$ 。

4) 古精见组今洪音前对立, 尖团不合流, 精组洪音前读 $[\mathrm{ts}] 、\left[\mathrm{ts}^{\mathrm{h}}\right] 、[\mathrm{~s}]$, 如: 资 $\left[\mathrm{ts}^{45}\right]$ 、坐 $\left[\mathrm{ts}^{\mathrm{h}} \mathrm{o}^{11}\right] 、 三\left[\mathrm{san}^{45}\right]$, 见 组洪音前读 $[\mathrm{k}] 、\left[\mathrm{k}^{\mathrm{h}}\right]$, 如: 高 $\left[\mathrm{kau}^{45}\right] 、$ 开 $\left[\mathrm{k}^{\mathrm{h}} \mathrm{ai}^{445}\right]$; 细 音前尖团合流腭化为 $[\mathrm{t} 6] 、\left[\mathrm{t}^{\mathrm{h}}\right] 、[6]$, 如: 酒 $\left[\mathrm{tciu}^{213}\right]$ 、 九 $\left[\mathrm{tciu}^{213}\right]$ 、就 $\left[\mathrm{tc}^{\mathrm{h}} \mathrm{iu}^{11}\right] 、$ 旧 $\left[\mathrm{tc}^{\mathrm{h}} \mathrm{iu} \mathrm{u}^{11}\right]$ 、洗 $\left[\mathrm{ci}^{213}\right]$ 、溪 $\left[\mathrm{ci}^{45}\right]$ 。

5) 古疑母与一、二等韵相拼, 今大部分读 $[\mathrm{y}]$, 如: 我 $\left[\mathrm{yo}^{45}\right]$ 、 牙 $\left[\mathrm{ya}^{453}\right]$; 与三、四等韵相拼, 大部分读 $[\mathrm{n}]$, 少数读

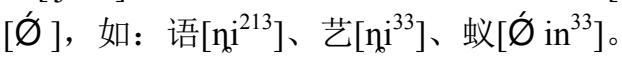

6) 古晓匣母与开口一、二等韵相拼, 今大部分读 $[\mathrm{h}]$, 如: 虾 $\left[\mathrm{ha}^{445}\right]$ 、河 $\left[\mathrm{ho}^{453}\right]$; 与三、四等韵相拼, 大部 分读[6], 如: 休 $\left[\mathrm{Giu}^{45}\right]$ 、歇 $\left[\mathrm{Gi \varepsilon}^{5}\right]$ 。古晓匣母与合口 一、二等韵相拼, 读零声母, 如: 灰 $\left[\varnothing \mathrm{uai}^{45}\right]$ 、坏 $[\varnothing$ $\left.\mathrm{uai}^{11}\right]$; 与合口三、四等韵相拼, 大部分读 [6], 如: 兄 ${ }_{2}\left[\operatorname{ciun}^{45}\right]$ 、熏 $\left[\operatorname{cin}^{45}\right]$ 。

\section{2. 韵母特点}

1) 石岗 (上坪) 方言无撮口呼, 遇摄合口三等泥精组字 韵母读 $[\mathrm{i}]$, 见组字韵母读 $[\mathrm{i}]$ 或 $[\mathrm{i} \varepsilon]$, 如: 女 $\left[\mathrm{ni}^{213}\right]$ 、语 $\left[\mathrm{ni}^{213}\right]$ 、鱼 $\left[\mathrm{ni}_{\mathrm{i}} \mathrm{e}^{453}\right]$ 。

2) 只有舌尖前元音[1], 无舌尖后元音[2]。

3) 通摄合口一、三等舒声韵, 大部分读[un]或[iun], 如: 东 $\left[\operatorname{tun}^{45}\right]$ 、送 $\left[\operatorname{sun}^{33}\right]$ 、熊 $\left[\operatorname{ciun}^{453}\right]$ 、嗅 $\left[\operatorname{ciun}^{33}\right]$, 合口三

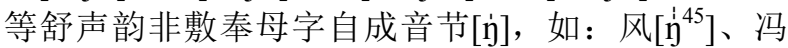
$\left[\mathfrak{y}^{143}\right]$ 、奉 $\left[\mathfrak{y}^{33}\right]$; 通摄合口一、三等入声韵大部分读 [u?], 如: 毒 $\left[\mathrm{th}^{\mathrm{h}} \mathrm{P}^{1}\right]$ 、竹 $\left[\mathrm{tu}^{5}{ }^{5}\right]$, 合口三等入声韵泥日见组部 分字读[iP]或 $[\mathrm{iu} P]$, 如: 菊 $\left[\mathrm{t} 6 \mathrm{i} \mathrm{P}^{5}\right]$ 、局 $\left[\mathrm{tci \textrm {P } ^ { 5 }}\right]$ 、肉 $\left[\mathrm{niu} \mathrm{P}^{5}\right]$ 、 绿[liu? ? $^{5}$ 。 


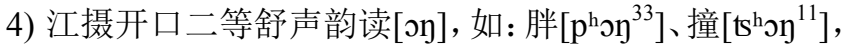
除疑母字读[ie?]外, 其它入声韵读[o?], 如: 剥[po? ${ }^{5}$ 、 桌[ $\left[\mathrm{to}^{5} \mathrm{P}^{5}\right] 、$ 乐音乐 $\left[\varnothing_{\mathrm{i}} \mathrm{i} ?^{5}\right]$ 。

5) 止摄三等韵不论开合, 大部分读 $[\mathrm{i}]$, 如: 被 $\left[\mathrm{p}^{\mathrm{h}} \mathrm{i}^{11}\right]$ 、 支 $\left[\mathrm{ti}^{45}\right]$, 止摄开口三等韵精知庄章组部分读 $[\varepsilon] 、[1]$ 、

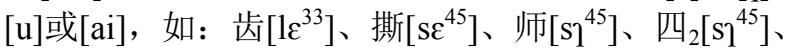
事 $\left[\mathrm{su}^{11}\right]$ 、紫 $\left[\mathrm{su}^{213}\right]$ 、篮 $\left[\mathrm{sai}^{45}\right]$; 止摄合口三等韵知庄 章泥组部分读[u]、[ui]或[ai], 如: 吹 $\left[\mathrm{t}^{\mathrm{h}} \mathrm{u}^{445}\right] 、$ 喂 $\left[\emptyset^{\mathrm{u}} \mathrm{ui}^{33}\right]$ 、 跪 $\left[\mathrm{k}^{\mathrm{h}} \mathrm{ui}^{453}\right.$ 、累 $\left[1 \mathrm{ai}{ }^{11}\right]$ 。

6) 遇摄合口一、三等韵大部分读 $[\mathrm{u}]$, 如: 普 $\left[\mathrm{p}^{\mathrm{h}} \mathrm{u}^{213}\right.$ 、 路 $\left[1 \mathrm{u}^{11}\right]$ 、猪 $\left[\mathrm{tu}{ }^{45}\right]$, 合口三等鱼虞韵精影组读 $[\mathrm{i}]$, 合 口三等韵见组个别字读[ic]或[ui], 如: 蛆 $\left[\mathrm{tg}^{\mathrm{h}} \mathrm{i}^{445}\right]$ 、语 $\left[\mathrm{ni}^{213}\right]$ 、鱼 $\left[\mathrm{ni}^{453}\right]$ 、句 $\left[\mathrm{kui}^{33}\right]$ 。

7) 蟹摄开口一、二等韵大部分读[ai], 如: 带 $\left[\mathrm{tai}^{33}\right]$ 、来 $\left[1 \mathrm{ai}^{453}\right]$ 、埋 $\left[\mathrm{mai}^{453}\right]$ 、界 $\left[\mathrm{kai}^{33}\right]$ 、鞋 $\left[\mathrm{hai}^{453}\right]$, 一等泰韵 帮组字读 $[\mathrm{i}]$, 如: 贝 $\left[\mathrm{pi}^{33}\right] 、$ 沛 $\left[\mathrm{p}^{\mathrm{h}} \mathrm{i}^{33}\right]$, 二等韵见组部 分读[a], 如: 佳 $\left[\mathrm{ka}^{45}\right]$ 、涯 $\left[\mathrm{na}^{453}\right]$; 开口三、四等韵大 部分读 $[\mathrm{i}]$, 三等韵章组个别读 $[\varepsilon]$, 四等韵匣母字读如

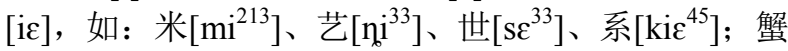
摄合口一等韵大部分读[i]或[ai], 个别读[uei]或[uai], 如: 最 $\left[\mathrm{tic}^{33}\right]$ 、队 $\left[\mathrm{ti}^{33}\right]$ 、背 $\left[\mathrm{pai}^{33}\right]$ 、外 $\left[\mathrm{yai}^{11}\right]$ 、碎 $\left[\mathrm{suei}^{33}\right]$ 、 块 $\left[\mathrm{kuai}^{33}\right]$; 合口二等韵读[uai], 如: 乘 $\left[\mathrm{kuai}^{45}\right]$ 、怪 $\left[\mathrm{kuai}^{33}\right]$, 佳韵和夫韵匣母字读[ua], 如: 话[Ǿ $\mathrm{ua}^{11}$ 、 画[ úua $^{11}$ 。

8) 殝摄开口一等韵读 $[\varepsilon n]$, 如: 跟 $\left[\mathrm{ken}^{45}\right]$ 、恩 $\left[\mathrm{n} \varepsilon \mathrm{n}^{45}\right]$; 檨 摄开口三等舒声韵读[in]或[ən], 如: 宾 $\left[\mathrm{pin}^{45}\right]$ 、亲 $\left[\mathrm{tg}^{\mathrm{h}} \mathrm{in}^{445}\right]$ 、真 $\left[\tan ^{45}\right]$ 、诊 $\left[\tan ^{213}\right]$, 入声韵读 $[\mathrm{it}]$ 或 $[\varepsilon ?]$, 如: 笔 $\left[\mathrm{pit}{ }^{5}\right]$ 、七 $\left[\mathrm{tt}^{\mathrm{h}} \mathrm{it}^{5}\right]$ 、实 $\left[\mathrm{s \varepsilon} \mathrm{P}^{1}\right]$ 、侄 $\left[\mathrm{t}^{\mathrm{h}} \varepsilon^{1}\right]$; 臻摄合 口一等舒声韵大部分读[ən], 见晓组个别字读[un],

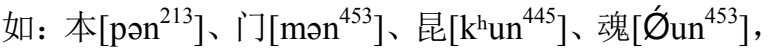

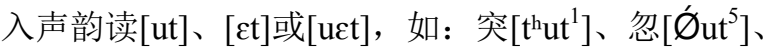
不 $\left[\mathrm{p \varepsilon t} t^{5}\right]$ 、骨 $\left[\mathrm{ku \varepsilon t} t^{5}\right]$; 合口三等舒声韵大部分读[ən]或 [in], 如: 轮 $\left[12 n^{453}\right]$ 、分 $\left[\operatorname{von}^{45}\right] 、$ 菌 $\left[\operatorname{tin}^{45}\right] 、$ 军 $\left[\operatorname{tcin}^{45}\right]$ 、 春 $\left[\mathrm{t}^{\mathrm{h}} \mathrm{n}^{445}\right]$, 合口三等入声韵读[u?]、[o?]或[i?], 如: 物 $\left[\varnothing_{\mathrm{u}} ?^{5}\right] 、$ 佛 $\left[\mathrm{vo}^{1}\right] 、$ 屈 $\left[\varnothing_{\mathrm{i}} ?^{5}\right]$ 。

9) 山摄开口一、二等舒声韵大部分读[an], 一等韵见晓 组读 $[\mathrm{on}]$, 二等韵明母字读 [on], 如: 丹 $\left[\mathrm{tan}^{45}\right]$ 、盼 $\left[\mathrm{p}^{\mathrm{h}} \mathrm{an}^{33}\right] 、 干\left[\mathrm{kon}^{45}\right]$ 、看 $\left[\mathrm{k}^{\mathrm{h}} \mathrm{on}^{33}\right]$ 、慢 $\left[\mathrm{mən}^{11}\right]$; 开口一、 二等入声韵大部分读 [at], 一等韵见晓组读[ot], 二等 韵明母字读 $[\varepsilon ?]$, 如: 达 $\left[\mathrm{tat}^{5}\right]$ 、辣 $\left[\mathrm{lat}^{1}\right]$ 、杀 $\left[\mathrm{sat}^{5}\right]$ 、渴 $\left[\mathrm{k}^{\mathrm{h}} \mathrm{ot}^{5}\right] 、$ 抹 $\left[\mathrm{m \varepsilon} \mathrm{P}^{5}\right]$; 开口、三四等舒声韵除知章组、 日母字读[an]外, 其它舒声韵读[izn], 如: 缠 $\left[\mathrm{t}^{\mathrm{h}} \mathrm{a} \mathrm{n}^{453}\right]$ 、 然 $\left[\mathrm{lan}^{453}\right]$ 、面 $\left[\mathrm{mi \varepsilon n}^{11}\right] 、$ 片 $\left[\mathrm{p}^{\mathrm{h}} \mathrm{i} \varepsilon \mathrm{n}^{33}\right]$; 开口三、四等入 声韵除知章组、日母字读[ct]外, 其它入声韵读[iet], 如: 撤 $\left[\mathrm{st}^{\mathrm{h}} \varepsilon \mathrm{t}^{5}\right]$ 、舌 $\left[\mathrm{sct}^{5}\right]$ 、别 $\left[\mathrm{p}^{\mathrm{h}} \mathrm{i \varepsilon t} \mathrm{t}^{\mathrm{l}}\right]$ 、切 $\left[\mathrm{tg}^{\mathrm{h}} \mathrm{i} \varepsilon \mathrm{t}^{5}\right]$; 山摄 合口一等舒声韵大部分读[on], 帮组读[en], 见晓组 读 $[\mathrm{u} \varepsilon \mathrm{n}]$, 如: 端 $\left[\operatorname{ton}^{45}\right]$ 、短 $\left[\operatorname{ton}^{213}\right]$ 、半 $\left[\mathrm{pen}^{33}\right]$ 、满 $\left[\mathrm{men}^{213}\right]$ 、

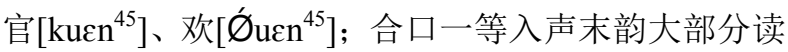

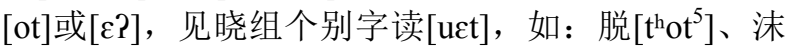

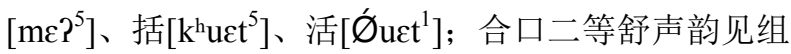
读[uan], 庄组读[on], 如: 鲤 $\left[\mathrm{kuan}^{45}\right] 、$ 幻 $\left[\right.$ Ǿuan $\left.^{11}\right]$ 、
门 $\left[\mathrm{son}^{45}\right]$ 、篡 $\left[\mathrm{s}^{\mathrm{h}} \mathrm{on}^{33}\right]$, 合口二等入声韵读[uat], 如: 滑 $\left[\right.$ $\left._{\text {uat }}{ }^{1}\right]$ 、挖 [Ǿuat $\left.{ }^{5}\right]$; 合口三、四等舒声韵大部分读

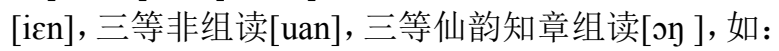
恋 $\left[n_{i \varepsilon n}{ }^{11}\right]$ 、县 $\left[\operatorname{cicn}^{33}\right]$ 、反 $\left[\varnothing_{u a n}{ }^{213}\right]$ 、烦 $\left[\varnothing_{\text {uan }}{ }^{453}\right]$ 、转 $\left[\operatorname{ton}^{33}\right]$ 、船 $\left[\operatorname{son}{ }^{453}\right]$; 山合口三、四等入声韵大部分读

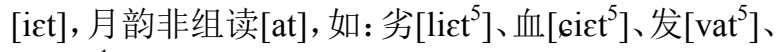
罚 $\left[\mathrm{vat}^{1}\right]$ 。

10)效摄开口一等韵读 [au], 如: 宝 $\left[\mathrm{pau}^{213}\right] 、$ 扫 $\left[\mathrm{sau}^{213}\right]$, 二、三、四等韵读 $[\mathrm{iau}]$ 或 $[\mathrm{au}]$, 如: 挑 $\left[\mathrm{t}^{\mathrm{h}} \mathrm{iau}^{445}\right]$ 、闹 $\left[\mathrm{lau}^{11}\right]$ 、 烧 $\left[\mathrm{sau}^{45}\right] 、$ 浇 $\left[\mathrm{kau}^{45}\right]$ 。

11)果摄开口一等韵端精组读 [uo], 见组读 $[\mathrm{o}]$, 泥组部 分读 $[\mathrm{a}]$ 或 $[\varepsilon]$, 如: 多 $\left[\mathrm{tuo}^{45}\right]$ 、左 $\left[\mathrm{suo}^{213}\right]$ 、挪 $\left[1 \mathrm{or}^{453}\right]$ 、 我 $\left[\mathrm{yo}^{453}\right]$ 、阿 $\left[\mathrm{na}^{45}\right]$ 、那 $\left[\mathrm{he}^{445}\right]$; 开、合口三等戈韵读

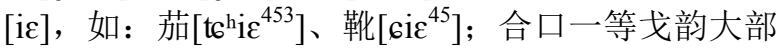
分读 $[\mathrm{o}]$, 精晓组部分读 [uo], 见组少数读 [u $\varepsilon]$, 如: 波 $\left[\mathrm{po}^{45}\right]$ 、椭 $\left[\mathrm{t}^{\mathrm{h}} \mathrm{o}^{213}\right]$ 、锁 $\left[\mathrm{suo}^{213}\right]$ 、过 $\left[\mathrm{kuo}^{33}\right] 、$ 果 $\left[\mathrm{ku} \varepsilon^{33}\right]$ 。

12)假摄开口二、三等韵读[a]或 [ia], 三等韵章组部分读 $[\varepsilon]$, 如: 巴 $\left[\mathrm{pa}^{45}\right]$ 、拿 $\left[\mathrm{la}^{453}\right]$; 姐 $\left[\mathrm{tcia}^{213}\right]$ 、遮 $\left[\mathrm{ta}^{45}\right]$ 、蛇 $\left[\mathrm{sa}^{453}\right]$ 、者 $\left[\mathrm{se}^{213}\right]$; 合口二等韵大部分读 $[\mathrm{a}]$, 见晓组 部分读[ua], 如: 俊 $\left[\mathrm{sa}^{213}\right]$ 、瓜 $\left[\mathrm{kua}^{45}\right]$ 、别 $\left[\mathrm{kua}^{213}\right]$ 、 瓦 $\left[\mathrm{ya}^{213}\right]$ 。

13)宕摄开口一等舒声唐韵大部分读[oy], 入声铎韵读 [o?], 如: 帮 $\left[\mathrm{pon}^{45}\right]$ 、当 $\left[\mathrm{ton}^{45}\right]$ 、薄 $\left[\mathrm{p}^{\mathrm{h}} \mathrm{P}^{1}\right]$ 、膜 $\left[\mathrm{mor}^{5}\right]$; 宕摄开口三等舒声阳韵大部分读[ion ], 知庄章组和

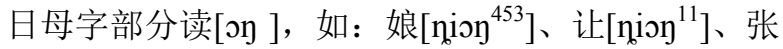
$\left[\operatorname{ton}^{45}\right]$ 、账 $\left[\operatorname{tgn}^{33}\right]$; 开口三等入声药韵读[ie?]、[io?]

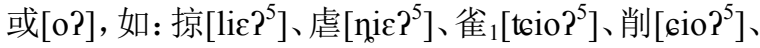
勺 $\left[\mathrm{soP}^{1}\right]$ 。

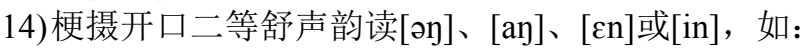
彭 $\left[\mathrm{p}^{\mathrm{h}} ә \eta^{453}\right]$ 、棚 $\left[\mathrm{p}^{\mathrm{h}} \partial \eta^{453}\right]$ 、冷 $\left[1 \mathrm{lag}^{213}\right]$ 、撑 $\left[\mathrm{s}^{\mathrm{h}} \mathrm{a} \mathrm{y}^{45}\right]$ 、更 $\left[\mathrm{ken}{ }^{33}\right]$ 、幸 $\left[\operatorname{cin}^{33}\right]$ 、行 $\left[\operatorname{cin}^{453}\right]$; 开口二等入声韵读[a?]、 [o?]或 [E?], 如: 百 $\left[\mathrm{pa}^{5}\right]$ 、麦 $\left[\mathrm{ma}^{1}\right]$ 、额 $\left[\mathrm{po}^{1}\right]$ 、核 $\left[\mathrm{ho} ?^{1}\right]$ 、 册 [ $\left[\mathrm{s}^{\mathrm{h}} \varepsilon \mathrm{P}^{5}\right]$ 、革 $\left[\mathrm{k} \varepsilon \mathrm{P}^{5}\right]$; 开口三、四等舒声韵读[in]或[ian], 如: 兵 $\left[\mathrm{pin}^{45}\right]$ 、挺 $\left[\mathrm{th}^{\mathrm{h}} \mathrm{in}^{213}\right]$ 、影 ${ }_{1}\left[\varnothing_{\mathrm{iag}}{ }^{213}\right]$ 、钉 $\left[\mathrm{tian}^{45}\right]$, 三等韵知章组读 $[ə n]$ 或 $[\mathrm{a}]$, 如: 贞 $\left[\tan ^{45}\right] 、$ 正 $\left[\tan ^{45}\right]$ 、 声 $\left[\operatorname{san}^{45}\right]$; 开口三、四等入声韵读[ia?]、[i?]和[a?], 如: 迹 $\left[\mathrm{tcia} 2^{5}\right]$ 、壁 $\left[\mathrm{pia} 2^{5}\right]$ 、惜 $\left[\mathrm{ci} ?^{5}\right]$ 、敌 $\left[\mathrm{ti} ?^{5}\right]$ 、只 $\left[\mathrm{ta}^{5}{ }^{5}\right]$ 、 石 [sa? $\left.{ }^{1}\right]$; 梗摄合口二等舒声韵见晓组读[uan], 如: 矿 $\left[\mathrm{k}^{\mathrm{h}} \mathrm{uan}^{33}\right]$ 、横 ${ }_{1}\left[\varnothing_{\mathrm{uan}}{ }^{453}\right]$; 合口二等入声韵匣母字读 [ua?], 如: 画[Ǿua? $\left.{ }^{5}\right]$ 、划[Ǿua? $\left.{ }^{5}\right]$; 合口三等庚韵读

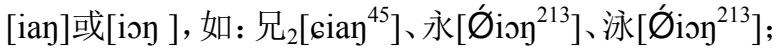
合口三等清韵、四等舒声韵读[in]或[ion ], 如: 倾 $\left[\mathrm{tg}^{\mathrm{h} i \mathrm{in}^{45}}\right]$ 、茧 $\left[\varnothing^{\mathrm{i}} \mathrm{in}^{453}\right]$ 、迴 $\left[\operatorname{tcioj}^{213}\right]$; 合口三等昔韵以 母入声读[i⿱龴⿵], 如: 疫[Ǿ $\left.\mathrm{i}{ }^{1}\right]$ 。

15) 曾摄开口一等舒声韵大部分读[ən]或[ $[\mathrm{en}]$, 帮组字读 $[ə \eta]$, 如: 崩 $\left[\mathrm{p} ə \eta^{45}\right]$ 、朋 $\left[\mathrm{p}^{\mathrm{h}} \mathrm{y}^{453}\right]$ 、曾 $\left[\mathrm{s}^{4}{ }^{45}\right]$ 、僧 $\left[\mathrm{s}^{4}{ }^{45}\right]$ 、 等 $\left[\operatorname{ten}^{213}\right]$ 、誊 $\left[\mathrm{t}^{\mathrm{h}} \varepsilon \mathrm{n}^{453}\right]$; 开口一等入声韵读 [ $[\mathrm{P}]$, 如: 刻 $\left[\mathrm{k}^{\mathrm{h}} \varepsilon^{3}\right] 、$ 北 $\left[\mathrm{p} \varepsilon ?^{5}\right]$; 开口三等舒声韵大部分读[in], 知章组和日母字读 [on], 如: 冰 $\left[\mathrm{pin}^{45}\right]$ 、兴 $\left[\mathrm{cin}^{33}\right]$ 、瞪 $\left[\tan ^{33}\right] 、$ 升 $\left[\operatorname{san}^{45}\right]$; 开口三等入声韵大部分读 $[\mathrm{i} ?]$, 知 庄章组读 $[\varepsilon ?]$, 如: 逼 $\left[\mathrm{pi}^{5}\right]$ 、力 $\left[\mathrm{li} 2^{5}\right]$ 、直 $\left[\mathrm{t}^{\mathrm{h}} \varepsilon ?^{1}\right]$ 、色 $\left[\mathrm{s \varepsilon}^{5}\right]$; 合口一等舒声韵读 $[\mathrm{un}]$, 如: 弘 $\left[\mathrm{xun}^{453}\right]$; 合 


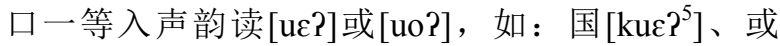
[Ǿ $\left.\mathrm{uo}^{1}{ }^{1}\right]$; 合口三等入声韵读[i?], 如: 域[Ǿ $\mathrm{i}^{1}{ }^{1}$ 。

16)流摄开口一等韵大部分读 $[\mathrm{au}]$ 或 $[\mathrm{u}]$, 见影组读 $[\mathrm{iau}]$, 如: 剖 $\left[\mathrm{p}^{\mathrm{h} a u^{445}}\right]$ 、母 $\left[\mathrm{mu}^{213}\right]$ 、贸 $\left[\mathrm{mau}^{11}\right]$ 、狗 $\left[\mathrm{kiau}^{213}\right]$ 、 藕 $\left[\mathrm{yiau}^{213}\right]$; 开口三等韵大部分读 $[\mathrm{iu}]$, 尤韵非庄组读 $[\mathrm{au}]$ 或 $[\mathrm{u}]$, 幽韵帮端组读 $[\mathrm{iau}]$, 如: 浮 $\left[\mathrm{p}^{\mathrm{h}} \mathrm{au}^{453}\right]$ 、谋 $\left[\mathrm{mau}^{453}\right]$ 、富 $\left[\mathrm{vu}^{33}\right]$ 、妇 $\left[\mathrm{vu}^{33}\right]$ 、刘 $\left[1 \mathrm{liu}^{453}\right]$ 、纠 $\left[\mathrm{tciu}^{45}\right]$ 、 扭 $\left[\mathrm{niu}^{213}\right]$ 、彪 $\left[\mathrm{piau}^{45}\right]$ 。

17)深摄开口三等舒声韵大部分读 $[\mathrm{in}]$, 知庄章组和日母 字读[ən]或[an], 如: 品 $\left[\mathrm{p}^{\mathrm{h}} \mathrm{in}^{213}\right] 、$ 林 $\left[\operatorname{lin}^{453}\right]$ 、沉 $\left[\mathrm{t}^{\mathrm{h}} \mathrm{n}^{453}\right]$ 、 枕 $\left[\tan ^{213}\right]$ 、秠 $\left[\operatorname{san}^{45}\right]$ 、婶 $\left[\operatorname{san}^{453}\right]$; 开口三等入声韵大 部分读 [it], 知庄章组读 $[\varepsilon \mathrm{t}]$, 日母字读 [ut], 如: 立 $\left[\mathrm{lit}^{1}\right] 、 习\left[\mathrm{cit}^{1}\right] 、$ 涩 $\left[\mathrm{sct}^{5}\right] 、$ 蛰 $\left[\mathrm{tsct}^{5}\right] 、 入\left[\mathrm{lut}^{1}\right]$ 。

18)咸摄开口一、二等和合口三等舒声韵大部分读 [an], 一等韵见晓组读[on], 二等韵匣母字读[iin], 如: 贪 $\left[\mathrm{than}^{\mathrm{h}}{ }^{45}\right.$ 、咸 $\left[\mathrm{han}^{453}\right]$ 、凡 $\left[\operatorname{van}^{453}\right]$ 、甘 $\left[\operatorname{kon}^{45}\right]$ 、敢 $\left[\operatorname{kon}^{213}\right]$ 、 岩 $\left[\varnothing_{i \varepsilon n^{453}}\right]$ 、衔 $\left[6 i \varepsilon n^{453}\right]$; 开口一、二等和合口三等 入声韵大部分读 [at], 一等韵见晓组读 [ot], 如: 答 $\left[\mathrm{tat}^{5}\right] 、$ 插 $\left[\mathrm{ts}^{\mathrm{h} a t^{5}}\right] 、$ 法 $\left[\mathrm{vat}^{5}\right] 、$ 盒 $\left[\mathrm{hot}^{1}\right] 、$ 磕 $\left[\mathrm{k}^{\mathrm{h}} \mathrm{ot}^{5}\right]$; 开口 三、四等舒声韵大部分读[ien], 三等韵章日组读[an], 如: 镰 $\left[1 i \varepsilon n^{453}\right]$ 、店 $\left[\operatorname{ticn}^{45}\right]$ 、占 $\left[\tan ^{33}\right]$ 、闪 $\left[\operatorname{san}^{213}\right]$; 开 口三、四等入声韵大部分读[iet], 三等入声韵章组读

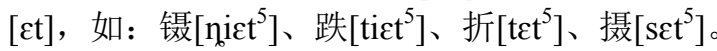

\section{3. 石岗（上坪）方言的文白异读}

文白异读是指同一个字在不同场域存在的不同读 音, 白读音代表某方言的土语, 而文读音则是方言吸收 的某一标准语成分, 从而向这一标准语靠拢。[10](第349 页)方言中, 文白异读现象表现在韵母、声母和声调上。 一般地认为, 它是语言(方言)接触的结果, 体现了方言 语音的不同层次; 是一种竞争关系, 而不是演变关系。 [11]

据统计, 南昌方言梗摄字、日母字和匣母字文白 读音数量较多。[12] (第154-216页) 石岗 (上坪) 方 言文白异读情况类似, 其部分字的白读音受普通话影 响, 呈式微之势; [13](第34页)文读音与普通话声韵相 同，仅声调有所差异，但总体上还处于 “白强文弱”的 阶段。此处对石岗 (上坪) 方言文白异读规律作些归 纳:

\subsection{1. 梗摄开口字的文白异读}

梗摄开口文白异读字数较多, 且其古今对应规律也较 整齐, 表现出较为完整的规律特点, 详见下表。

表2 梗摄开口字文白异读。

\begin{tabular}{llll}
\hline & 二等 & 三四等 & \\
\cline { 3 - 4 } & & 知章组 & 其他 \\
\hline 白读 & $\mathrm{a} / \mathrm{a}$ ? & $\mathrm{a} / \mathrm{a}$ ? & $\mathrm{ian} / \mathrm{ia} ?$ \\
文读 & $\varepsilon \mathrm{n} / \mathrm{\varepsilon t}$ & $\mathrm{on} / \varepsilon \mathrm{t}$ & $\mathrm{in} / \mathrm{it}$ \\
\hline
\end{tabular}

表中表明, 梗摄开口保留了入声 $[\mathrm{t}]$ 尾和喉塞韵尾, 入 声文读以 $[\mathrm{t}]$ 收尾, 白读以喉塞音[?]收尾; 开口舒声文读以 $[\mathrm{n}]$ 收尾, 白读以[n]收尾。[14]下面列举其文白异读例字,
一个字有两读的, 先列白读, 再列文读, 文读置于“||”之 后。例如:

坑: $\left[\mathrm{k}^{\mathrm{h} a \eta^{445}}\right]$ 挖 $\sim \|\left[\mathrm{k}^{\mathrm{h}} \varepsilon \mathrm{n}^{445}\right] \sim 人$

硬: $\left[\mathrm{gan}^{33}\right]$ 饭好 , 座

生: $\left[\mathrm{san}^{45}\right] \sim$ 饭, 认 $\|\left[\mathrm{s} ə^{45}\right] \sim$ 日, 活

争: $\left[\operatorname{san}^{45}\right] \sim$ 气, 莫 $\sim\left[\operatorname{ts}^{45}\right]$ 战 , 为国 光

省: $\left[\operatorname{san}^{213}\right] \sim$ 钱, 穴, 事 $\|\left[\operatorname{san}^{213}\right]$ 江西

病: $\left[\mathrm{p}^{\mathrm{h}} \mathrm{ia \eta}^{11}\right] \sim$ 人, 得,$\sim$ 情

正: $\left[\tan ^{45}\right] \sim$ 月里, 话 了说好了 $\|\left[\tan ^{33}\right] \sim$ 反, 事, 公

命: $\left[\operatorname{mian}^{11}\right]$ 算,$\sim 大$, 救 $\sim \|\left[\min ^{11}\right] \sim$ 案, 令

影: $\left[\varnothing_{\mathrm{ia \eta}^{213}}\right] \sim$ 子 $\|\left[\varnothing_{\mathrm{in}}{ }^{213}\right]$ 电 , 响

姓: $\left[\operatorname{cian}^{33}\right] \sim$ 张, 李 $\|\left[\operatorname{cin}^{33}\right]$ 贵 , 名

石: $\left[\mathrm{sa}^{1}\right] \sim$ 头, $\sim$ 灰 $\|\left[\mathrm{sct}^{1}\right] \sim$ 榴

百: $\left[\mathrm{par}^{5}\right]$ 一 $\|\left[\mathrm{pet}^{5}\right] \sim$ 姓, 货

格: $\left[\mathrm{kaP}^{5}\right] \sim$ 子 $\|\left[\mathrm{ket}^{5}\right]$ 及

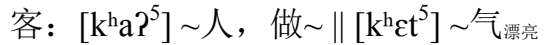

滴: $\left[\mathrm{tia}^{5}\right]$ 一子 $\|\left[\mathrm{tit}^{5}\right] \sim$ 水, $\sim$ 眼药水

\subsection{2. 日母字的文白异读}

假摄和止摄日母字, 读零声母的字没有文白之分, 如: 二、耳、儿、惹等; 其它摄的日母字, 文读音受今北方方 言影响, 声母读[1], [15]白读音是古音遗留[n]。日母字读 [n] 与细音[i]相拼, 白读[1]与洪音相拼, 因此其文白异读现 象主要表现在声母和韵母上面。下面列举日母字文白异读 的例字, 一个字有两读的, 先列白读音, 再列文读音, 文 读音列于“||”之后。例如:

染: $\left[\operatorname{lan}^{213}\right] \| \sim$ 色, 〜布, 传

人: $\left[\operatorname{nin}^{453}\right]$ 大, 老 家, 犯 , 家 $\|\left[1 ə n^{453}\right] \sim$ 参, 格, 〜品

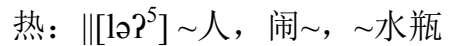

软: $\left[\right.$ nicn $\left.^{213}\right] \sim$ 骨头, 心 , 些 很软 $\|\left[\operatorname{luan}^{213}\right] \sim$ 弱, 柔

忍: $\left[\operatorname{nin}^{213}\right] \sim$ 住, 到 $\|\left[\operatorname{lon}^{213}\right] \sim 心, ~ \sim$ 受

认: $\left[\operatorname{nin}^{11}\right] \sim$ 得, 生, 账 $\|\left[\operatorname{lon}^{11}\right]$ 承

日: $\left[\mathrm{nit}^{5}\right] \sim$ 头, 明 \| [lot $\left.{ }^{5}\right]$ 生 , 记, 厉

让: [nion $\left.{ }^{11}\right] \sim 一 下 \|\left[1 \circ \eta^{11}\right]$ 迁 , 路

弱: \|[luo $\left.P^{5}\right]$ 虚 $\sim$, 小

肉: [niu $\left.{ }^{5}\right]$ 长 , 猪

闰: $\|\left[l^{1} n^{11}\right] \sim$ 月里

仁: $\|\left[1 ə n^{453}\right] \sim$ 慈, $\sim$ 义道德

\subsection{3. 匣母字的文白异读}

匣母一、二等韵中, 声母今一律读[h], 韵母为洪音; 其它四等字 (除蟹摄合口四等雯韵中的“惠、慧”读零声母、 合口韵外) 不论开合声母一律读[6], 韵母为细音。本文选 取匣母字文白现象较多的合口一、二等韵为例, 白读音声 母为零声母[自或 $[\mathrm{n}]$, 文读音声母为 $[\mathrm{v}]$ 或 $[\mathrm{h}]$ 。下面列举的 匣母字文白异读, 一个字两读的, 先列白读音, 再列文读 音，文读音放在“|”后，如：

和: $\|\left[\mathrm{ho}^{33}\right] \sim$ 尚, $\sim$ 气 $\left[\mathrm{vo}^{33}\right]$ 暖 , 热

划: [Ǿ $\left.\mathrm{ua}^{453}\right] \sim$ 算, 船, 线 $\|\left[\operatorname{Cu}^{33}\right]$ 线

胡: $\left[\varnothing \mathrm{u}^{453}\right] \sim$ 子, 〜老师

回: [Ǿ $\left.\mathrm{uai}^{11}\right] \sim$ 得回了礼 $\|\left[\mathrm{vi}^{453}\right] \sim$ 来, 家, 来来 


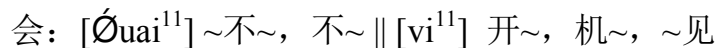

坏: [Ǿuai $\left.{ }^{11}\right] \sim$ 的, 好 , $\sim$ 人

话: [Ǿua $\left.{ }^{11}\right] \sim$ 事, 媒, 〜一句 $\|\left[\mathrm{va}^{11}\right] \sim$ 剧

丸: [Ǿuan $\left.{ }^{453}\right]$ 肉 子

活: [Ǿ $\left.\mathrm{uct}^{5}\right] \sim$ 的, 宝 $\|\left[\mathrm{v \varepsilon t}^{5}\right]$ 生

换: [Ǿuan $\left.{ }^{11}\right] \sim$ 成, 一下

还: [Ǿ $\mathrm{uan}^{453}$ ] 得回去回礼, 价, 魂, 借了要

环: [Ǿ $\left.\mathrm{uan}^{453}\right]$ 耳 $\sim \|\left[\mathrm{van}^{453}\right] \sim$ 境, 门

魂: $\|\left[\mathrm{van}^{453}\right]$ 叫, , 灵

黄: [Ǿu的 $\left.{ }^{453}\right] \| \sim$ 色, 昏, 生 很黄

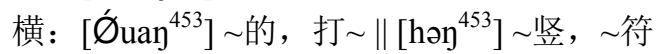

红: $\left[\mathfrak{j}^{453}\right] \sim$ 色, 鲜 , 领 $\|\left[\mathrm{h} \supset \mathrm{y}^{453}\right] \sim$ 军

\section{4. 声韵配合规律}

石岗 (上坪) 方言声韵配合除部分不循规蹈矩外，大 部分还是有着十分严整的规律性, 这在以下声韵配合表中 有所体现。

表3 声韵配合规律。

\begin{tabular}{|c|c|c|c|c|}
\hline 声母 & 韵呼 & 开口呼 & 齐齿呼 & 合口呼 \\
\hline 双唇音 & $\mathrm{p}, \mathrm{p}^{\mathrm{h}}, \mathrm{m}$ & + & + & 只拼u \\
\hline 唇齿音 & $\mathrm{V}$ & + & 只拼 $\mathrm{i}$ & \\
\hline 舌尖前音 & ts, ts ${ }^{\mathrm{h}}, \mathrm{s}$ & + & & + \\
\hline 舌尖中音 & $\begin{array}{l}\mathrm{t}, \mathrm{t}^{\mathrm{h}}, 1 \\
\mathrm{n}\end{array}$ & + & + & 只拼u \\
\hline 舌面音 & tc, $t c^{h}, 6$ & & $\begin{array}{l}\text { 穴拼1 } \\
+\end{array}$ & \\
\hline 舌根音 & $\mathrm{k}, \mathrm{k}^{\mathrm{h}}, \mathrm{n}$ & $\begin{array}{l}+ \\
+\end{array}$ & 只拼i $\mathrm{E}$ & + \\
\hline 喉音 & h & + & & \\
\hline 零声母 & Ǿ & + & + & + \\
\hline
\end{tabular}

声韵配合规律说明:

1) 石岗(上坪)方言中无撮口呼, 双唇音可与开口呼、 齐齿呼和合口呼韵母相拼, 如: 宝 $\left[\mathrm{pau}^{213}\right]$ 、笔 $\left[\mathrm{pit}^{5}\right]$ 、 布 $\left[p u^{33}\right]$ 、爬 $\left[p^{h} a^{453}\right]$ 、皮 $\left[p^{h i^{453}}\right]$ 、普 $\left[p^{h} u^{213}\right]$ 、马 $\left[\mathrm{ma}^{213}\right]$ 。

2) 唇齿浊擦音 $[v]$ 与齐齿呼韵母 $[\mathrm{i}]$ 相拼, 大部分是与三 等韵非组字组合, 如: 肥 $\left[\mathrm{vi}^{453}\right] 、$ 非 $\left[\mathrm{vi}^{45}\right] 、$ 味 $\left[\mathrm{vi}^{11}\right]$ 。

3) $[\mathrm{h}]$ 不与合口呼相拼, 若与之组合则多变为零声母, 大部分来源于古匣母, 如: 会 $\left[\emptyset_{u a i}{ }^{11}\right]$ 、胡 $\left[\emptyset^{4}{ }^{453}\right]$ 。

4) $[\mathrm{t} c] 、\left[\mathrm{t}^{\mathrm{h}}\right] 、[\mathrm{c}] 、[\mathrm{n}] 、[\mathrm{n}]$ 只与齐齿呼韵母相拼, 不 拼开口呼和合口呼, 如: 精 $2\left[\operatorname{tcin}^{45}\right]$ 、青 $\left[\operatorname{tcian}^{445}\right]$ 、 想 $\left[\operatorname{cioj}^{213}\right]$ 、年 $\left[\operatorname{nicn}^{453}\right]$ 。

5) $[\mathrm{k}] 、\left[\mathrm{k}^{\mathrm{h}}\right]$ 可与齐齿呼韵母相拼, 只是字数较少而已, 如: 锯 $\left[\mathrm{kic}^{33}\right] 、 \square_{\text {锹断 }}\left[\mathrm{ki}^{\mathrm{hi}} \varepsilon^{453}\right]$ 。

6) 零声母可与齐齿呼、合口呼和开口呼[2]韵母组合,

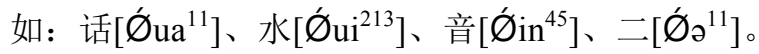

\section{5. 结语}

概而言之, 以上对石岗 (上坪) 方言音系及其特点作 了较为全面而系统地分析阐述, 由此可见石岗 (上坪) 方
言既有赣方言音韵的共同特征, 又有自身独具的特点, 它 保留了入声 $[\mathrm{t}]$ 尾和喉塞韵尾, 仅是分化条件有所不同而已。 就其分化条件而言, 咸、深、山、臻几摄韵母存有 $[\mathrm{t}]$ 尾, 宕、江、曾、梗、通则留有喉塞韵尾, 不过由于它们还处 在不断演变过程之中, $[\mathrm{t}]$ 尾已有向喉塞韵尾变化的趋势。 另外, 石岗 (上坪) 方言的文白异读特点鲜明, 而且还有 文白混读的情况, 当然其文白音正处于一个逐渐消退的过 程之中。一段时间以来, 人们的交际语呈现出这样一大演 变趋势: 语言交际由原来的单言 (方言) 现象变为双言并 存 (方言与共同语), 继而又朝着单言 (共同语)方向发 展, 即使一个家庭也是如此。[16]毫无疑问, 石岗 (上坪) 方言如同其它方言或南昌方言一样, 也渐渐朝着趋普变异 的方向变化。[17]（第137页）一句话, 石岗 (上坪) 方言 在其音系或音韵相配规律上有其独特之处, 但随着社会的 发展, 它也在发生着变化, 其原有的特点正渐次消失。

\section{致谢}

基金项目: 中国江西省学位与研究生教育教学改革研 究项目“揭开“绝学”面纱, 继承传统文化一一关于研究生课 程《音韵学》教改问题的几点思考”（JXYJG-2014-038） 成果之一。

\section{参考文献}

[1] Wan Yali.On the unique phenomenon of Nanchang dialect [D]. Dalian University of Technology, 2011.

[2] 谢留文.江西省的汉语方言[J].方言,2008(02).

[3] 陈昌仪.新建方言音系[J].抚州师专学报,1991(02).

[4] Yu Chunping. A comparative study on the vocabulary of Xinjian dialect in Jiangxi Province [D]. Hunan Normal University, 2014.

[5] 中国社会科学院语言研究所.方言调查字表[M].商务印书 馆,2018.

[6] 卢继芳. 赣语昌都片语音研究[D].湖南师范大学博士论 文,2016.

[7] 颜森.江西方言的声调[J].江西师范大学学报,1988(03).

[8] 孙宜志.江西赣方言语言研究[M]. 语文出版社, 2007.

[9] 项梦冰.赣语古全浊声母今读浊音的类型 [J]. 语言学论 从, 2013(01).

[10] 徐通铭.历史语言学[M].商务印书馆,1991.

[11] 谢留文.汉语方言研究七十年[J].方言,2019(03).

[12] Lin Songyu.On the literary and colloquial pronunciations of Chinese dialects [D]. Fujian Normal University, 2011.

[13] Tu Na.A study on the colloquial sayings of Nanchang dialect [D]. Nanchang University, 2014. 
[14] 熊正辉.南昌方言的文白读[J].方言,1985(03).

[15] 左国春.江西临川方言的文白异读[J].文教资料,2013(16).

[16] 刘 群.家庭语言规划和语言关系[J].江西师范大学学报(哲 社科), 2017（06）.
[17] Chu Qianwen. An experimental study on the phonological system of Nanchang dialect [D]. Nanjing Normal University, 2018. 